\title{
Bone health in patients with multiple sclerosis relapses
}

Olwen Murphy MRCPI,,${ }^{1,2}$ Michael S Zandi PhD MRCP,,${ }^{1,3}$ Nitzan Lindenberg, ${ }^{1}$ Elaine Murphy MRCPI FRCPath, ${ }^{4}$ Jeremy Chataway PhD FRCP. ${ }^{1}$

${ }^{1}$ Queen Square Multiple Sclerosis Centre, Department of Neuroinflammation, UCL Institute of Neurology, University College London and National Hospital for Neurology and Neurosurgery, University College London Hospitals NHS Foundation Trust, London, United Kingdom

${ }^{2}$ Department of Neurology, Cork University Hospital, Wilton, Cork, Ireland

${ }^{3}$ Department of Neurology, Addenbrooke's Hospital, Cambridge University Hospitals NHS Foundation Trust, Hills Road, Cambridge, United Kingdom

${ }^{4}$ Charles Dent Metabolic Unit, University College London and National Hospital for Neurology and Neurosurgery, University College London Hospitals NHS Foundation Trust, London, United Kingdom

Corresponding author: Dr. Olwen Murphy, Cork University Hospital, Wilton, Ireland. Telephone: 00353861049606. Email: murphy.olwen@gmail.com.

Word count: 2666 (excluding abstract, tables and references)

\section{Abstract word count: 249}

Keywords: multiple sclerosis, metabolic bone disease, bone mineral density, vitamin D. 


\section{ABSTRACT}

Objectives: To evaluate the bone health and vitamin D levels of a cohort of patients with relapses of multiple sclerosis (MS) and to propose an algorithm for the management of bone health in this patient group.

Methods: We prospectively studied 56 consecutive patients from our acute relapse clinic. 3 patients were excluded from analysis as they were not deemed to have experienced an acute MS relapse. Bone health was assessed with vitamin D levels and Dual Energy X-ray Absorptiometry (DEXA) scanning (10 patients failed to attend for DEXA). Statistical analyses were used to compare groups and identify predictive variables. A review of the literature led to a proposed management protocol.

Results: Pre-relapse the baseline EDSS was $\leq 6.5$ in all subjects, and $<4.0$ in the majority $(66 \%)$. Most received corticosteroids. 51\% had low bone mineral density (BMD) as defined by a T-score less than 1.0 on DEXA scanning. Three were osteoporotic (T-score less than -2.5$)$. Thirty one of fifty (62\%) subjects were Vitamin D deficient $(25(\mathrm{OH}) \mathrm{D}$ less than $50 \mathrm{nmol} / \mathrm{L})$. A range of variables, including previous corticosteroid usage, were not significantly predictive of reduced BMD.

Conclusions: There was a high frequency of both low BMD and Vitamin D deficiency in this cohort of relatively young and largely ambulatory patients experiencing MS relapses. Current tools, such as the WHO FRAX algorithm, are inadequate in assessing bone status and fracture risk in this patient group, predominantly as they are focused on older age groups. We propose a simple clinical management algorithm. 


\section{INTRODUCTION}

Metabolic bone disease is under-recognised and under-treated in multiple sclerosis (MS). There is a lack of evidence and guidance for the recognition and treatment of metabolic bone disease, particularly in patients with relapsing-remitting MS (RRMS) who may frequently be exposed to high doses of corticosteroids. Patients with MS (PwMS) have higher rates of fractures than the general population[1-2] and reduced bone mineral density (BMD) compared to healthy controls[3]. Increasing neurologic impairment as categorised by the Kurtzke Expanded Disability Status Scale (EDSS)[4] is a key factor contributing to osteopenia and osteoporosis in PwMS.[5-7] However, while poor bone health is common in many chronic neurological diseases, reduced BMD has been identified even in the early stages of MS when patients are fully ambulatory and EDSS is low.[8] High-dose methylprednisolone (intravenous or oral, 3-5g) up to 2-3 times/year is a common treatment for relapses of MS, and is effective in reducing relapse duration.[9] Whilst long-term steroid treatment is a clear risk factor for osteopenia (and osteoporosis) as recognised in the FRAX algorithm; the position is much less clear for pulses of steroid which last for under a week. Short-term corticosteroid therapy has been shown to affect the biochemical markers of bone turnover in PwMS[10], but the evidence for corticosteroid-induced osteopenia in this group is conflicting.[5,11-12]

Management of bone health in this population is challenging, as it is difficult to stratify fracture risk. The WHO FRAX calculator (www.shef.ac.uk/FRAX/) is a tool for predicting risk of fracture, however it has a number of limitations in the setting of MS. It is validated for a general population cohort over the age of 40[13], while in a typical relapse clinic the average age is 40 and women are pre-menopausal[14]. Steroids are only included as a risk factor in the FRAX calculation if the dose is at least $5 \mathrm{mg} /$ day for more than 3 months, MS is not included as an independent risk factor, and it does not take into account Vitamin D deficiency which is associated with MS[15] and an important modifier of bone health. An alternative, the recent Qfracture tool, includes a wider age range and additional risk factors than the FRAX,[16] however even with this tool the projection of the risk of fracture in this specific population is limited and likely to be an underestimation.[17] Bazelier et al. 
recently described a clinical risk score for the estimation of fracture risk for PwMS which is likely to give a better estimation of fracture risk in this specific population.[18] However this scoring system needs further validation before routine use in a clinical context.

To examine these issues, we wanted to determine the bone status of a sequential cohort of patients attending an acute MS relapse clinic, who received high-dose steroid treatment. We aimed to identify any predictive variables for reduced BMD and create a simple management algorithm to improve quality of clinical care for this patient group.

\section{METHODS}

The National Hospital of Neurology and Neurosurgery MS relapse service has been described before,[19] but in brief, PwMS are tele-triaged by an MS-trained healthcare professional and then, if a significant relapse is occurring, proceed to a weekly ambulatory outpatient clinic. After assessment, if clinically indicated, a standard steroid regime of intravenous methylprednisolone $1 \mathrm{~g} / \mathrm{day}$ for 3 days is used along with multi-disciplinary therapy input.

We included 56 consecutive patients from our clinic in this quality improvement study. Following medical assessment, 3 patients were deemed to have symptoms which were not compatible with an acute relapse and were excluded from statistical analysis. Demographic information was recorded for all patients, as well as details of MS type and duration, current medications including calcium/vitamin D supplementation, treatment with corticosteroids in the preceding two years (intravenous or oral form) and both pre-relapse and in-relapse EDSS. Lifestyle variables relevant to bone health were also recorded, including body mass index (BMI), smoking status, alcohol consumption, menopausal status and history of fragility fractures. 
Blood tests (total 25-hydroxyvitamin D [25(OH)D] thyroid function tests, parathyroid hormone, renal, liver and bone profiles) were taken at the time of clinic attendance (between January and April). 25hydroxyvitamin D2 and 25-hydroxyvitamin D3 were measured using liquid chromatography tandem mass spectrometry (LC-MS/MS), and these were combined to give total 25(OH)D. 25(OH)D levels below $7 \mathrm{nmol} / \mathrm{L}$ were arbitrarily scored as $5 \mathrm{nmol} / \mathrm{L}$. Dual energy X-ray absorptiometry (DEXA) scanning was arranged within three months of attendance at clinic; DEXA T-scores and Z-scores were obtained for the hip, neck of femur and lumbar spine. T-scores compare BMD of an individual with that of a young healthy population, with osteoporosis defined as SD $\leq-2.5$ from the mean, and osteopenia as $\mathrm{SD} \leq-1.0$ from the mean. A Z-score compares an individual's BMD to that of an age and sex matched population. 10 subjects failed to attend for scheduled DEXA scans, and comparing this group to the 43 subjects who did attend for DEXA, there were no significant differences in terms of gender, age, MS duration or EDSS.

FRAX scores were calculated using the online WHO FRAX Calculator.[20] For patients under 40 the model calculates the probability of a fracture at age 40 (likely to result in an overestimation of fracture risk in younger subjects).

Comparisons between the groups were made with Student's t test for continuous variables and Fisher's exact test for categorical variables. Logistic regression was used to identify any predictive variables for osteopenia/osteoporosis, and linear regression to identify the lowest measured $\mathrm{T}$ score in an individual. BMI, age, sex, vitamin D levels, EDSS pre- and in-relapse, FRAX scores without bone density, smoking status and previous steroid exposure were entered as variables. Patients consented to inclusion and ethical approval was not required for this study as it was a service evaluation.[21] 


\section{RESULTS}

The cohort characteristics are described in Table 1. The mean age of all subjects was 39.6 years and the majority of patients $(66 \%, 95 \%$ C.I. 52 to $80 \%)$ were fully ambulatory pre-relapse with an EDSS of <4.0. The maximum pre-relapse EDSS of any subject was 6.5 , and the majority ( $87 \%$, $95 \%$ C.I. 78 to $96 \%$ ) were classified as having relapsing remitting MS (RRMS). In-relapse EDSS was $\geq 4.0$ in the majority of subjects $(80 \%$, 95\% C.I. 68 to $92 \%)$, with 21 subjects moving from being fully ambulatory (EDSS <4.0) to having some mobility limitation (EDSS $\geq 4.0$ ) as a result of relapse. A sensitivity analysis was performed assigning EDSS levels $<4$ as either 0 or 4 and there was no significant difference in the outcome of statistical analysis. Mean 25(OH)D was $45.6 \mathrm{nmol} / \mathrm{L}$ (range 5.0 to $162 \mathrm{nmol} / \mathrm{L}$ ). $96 \%$ of patients were treated with steroids as a result of their attendance in the MS relapse clinic.

Table 2 shows the 43 subjects with DEXA results (10 did not attend their appointment). DEXA scans were performed at mean 58 days after corticosteroid treatment. $22(51 \%)$ patients were osteopenic (3 of whom were osteoporotic). Two of the 3 osteoporotic patients were post-menopausal women. Thirty-one of $50(62 \%, 95 \%$ C.I. 49 to $75 \%)$ patients were vitamin D deficient based on the $50 \mathrm{nmol} / \mathrm{L}$ cut-off recommended by the United States Endocrine Society.[22] Prevalence of Vitamin D deficiency in our cohort was higher than prevalence in the British population (range $3.2 \%$ to $46.6 \%$ depending on seasonal variation and cut-offs of either $25 \mathrm{nmol} / \mathrm{L}$ or $40 \mathrm{nmol} / \mathrm{L})$.[23] 25(OH)D levels were measured between January and April and are likely to reflect some seasonal variation $(25(\mathrm{OH}) \mathrm{D}$ serum levels reach an annual trough in February).[24] While methods of adjusting Vitamin D for month and season have been described,[25] this standardisation method is not routinely used in clinical practice and cut-offs for sufficiency/deficiency apply year-round. However, there is a lack of global consensus on exact serum cut-offs for vitamin D deficiency/insufficiency.[26] 
Table 3 compares patients with normal BMD to those with osteopenia/osteoporosis. None of the variables of 25(OH)D levels, previous corticosteroid usage and pre-relapse EDSS were significantly different between these groups. There was no significant difference in FRAX scores pre-DEXA scanning comparing patients with normal BMD versus osteopenia/osteoporosis. The only significant difference between the groups resulted from the comparison of FRAX scores post-DEXA scanning.

Using logistic regression, no variable significantly predicted the binary outcome of osteoporosis/osteopenia versus normal bone density. Similarly, standard linear regression found no significant predictors of lowest DEXA T-score in this study.

\section{DISCUSSION}

Significantly reduced BMD (approximately half of all patients [51\%, 95\% C.I. 36.1 to 65.9]) and 25(OH)D deficiency (approximately two thirds of all patients [62\%, 95\% C.I. 48.6 to 75.4$]$ ) were common in this sequential cohort of about 50 patients assessed following an MS relapse, despite the young average age (majority pre-menopausal) and low levels of pre-relapse disability (66\% EDSS $<4.0,95 \%$ C.I. 52 to $80 \%$ ). However statistical analysis failed to reveal any significant differences between those with normal BMD and those with osteopenia in terms of: $25(\mathrm{OH}) \mathrm{D}$ levels, relapse frequency or disability level (either pre-relapse or in-relapse EDSS).

It has been difficult to elucidate the reason for reduced bone density in the early stages of MS. Various studies have shown heterogeneity of predictive variables. Kirbas et al.[27] and Moen et al.[28] suggest that this points to a shared aetiology of MS and osteopenia/osteoporosis. Sioka et al. have suggested that the inflammatory process with relapsing MS may be detrimental to bone status[3], which is supported in the literature relating to rheumatic disease.[29] This hypothesis is supported by the disproportionately low BMD we have demonstrated in our study of young ambulatory patients with active MS. Finally, the evidence for an epidemiological association between MS and Vitamin D 
deficiency continues to accrue[30], and vitamin D levels are an important long-term modifier of bone health.[31]

Our study shows that the frequency of steroid course over the preceding 2 years did not influence BMD, and supports other studies demonstrating no detrimental impact of short-term high-dose steroids.[12] Indeed, glucocorticoid-induced osteoporosis is only considered a risk in those patients who receive at least $5 \mathrm{mg}$ Prednisolone (or equivalent dose of other glucorticoids) for at least 3 months.[32] However there is increasing evidence that the impact of steroids on bone health extends beyond BMD. Patients on long term steroids experience fractures at a higher BMD than controls [33] and steroids have been shown to have a negative impact on bone modeling and architecture that is not detected in standard DEXA scanning. [34,35] Further longitudinal studies are required to examine the influence of high-dose corticosteroid use on long-term outcome measures (including fracture rates) in the MS population.

The question arises as to how to assess the bone health of patients attending with an MS relapse, if it had not been previously or recently examined. From this data, one approach would be to measure the Vitamin D and BMD using DEXA of all patients attending with a relapse, since their risk appears to be so high. However, considering the young age of this population and the resource utilisation this would require, we advocate a more conservative approach to identify patients in need further investigation.

Considering the above, we suggest here an algorithm to manage the inter-related issues of $25(\mathrm{OH}) \mathrm{D}$ deficiency and reduced BMD (Figure 1). Initial assessment includes evaluation of lifestyle factors along with blood tests relevant to bone health, including serum $25(\mathrm{OH}) \mathrm{D}$ on a yearly basis. All patients should be advised regarding conservative measures to maintain bone density. 


\section{Vitamin D Deficiency}

Patients should be offered Vitamin D supplementation if their serum level is deficient $(<50 \mathrm{nmol} / \mathrm{L})$. In particular as table 1 shows, less than $20 \%$ of this cohort was taking vitamin D/calcium supplements, before the high prevalence of reduced BMD was identified from this study. While $50 \mathrm{nmol} / \mathrm{L}$ is considered a lower cut-off for Vitamin D deficiency, $70-80 \mathrm{nmol} / \mathrm{L}$ should be targetted as the optimal serum level for bone health[37,38]. To achieve target levels, doses up to $10000 \mathrm{IU}$ per day appear to be safe.[39] We recommend supplementation with Cholecalciferol (Vitamin D3) at a dose of 10000 or 20000 units weekly which should be sufficient to achieve target serum Vitamin D levels. Calcium should be supplemented with $1 \mathrm{~g}$ Calcium Carbonate daily if there is dietary deficiency.[40] Serum calcium should be measured after 1 month, as Vitamin D supplementation can unmask hyperparathyroidism.[41] We recommend reassessing Vitamin D levels after 6 months of supplementation, when a steady state is achieved.[41] Dosing can be adjusted if necessary at this point, including dose reduction if required (in view of recent evidence that $25(\mathrm{OH}) \mathrm{D}$ levels greater than $120 \mathrm{nmol} / \mathrm{L}$ are associated with increased all-cause mortality [42]). Once a steady state of sufficiency is achieved, draft guidelines from the National Osteoporosis Society indicate that routine monitoring of 25(OH)D is unnecessary[41], however it would seem prudent to monitor calcium levels annually, in case of hypercalcaemia.

\section{DEXA Scanning}

Next we consider which patients require formal DEXA scanning. As highlighted by Dobson et $a l .,[40]$ there is a lack of disease specific evidence or guidelines for the monitoring of bone health in this cohort, but a practical and proactive approach should be employed for early identification of those at increased risk of fractures. DEXA scanning is a non-invasive imaging method with a very low radiation dose (approximately 2.5 microSv).[43] Post-menopausal women are at highest risk of osteoporosis, and we recommend that a DEXA scan is included in initial assessment. For the rest of 
the patient population (men and pre-menopausal women), we would recommend proceeding to DEXA scan if mobility is impaired (i.e. pre-relapse EDSS $\geq 4.0$ ) or falls risk is high. For patients without these additional risks, we consider the FRAX or QFracture tools to be a useful adjunctive assessment. We have demonstrated that FRAX scores calculated pre-DEXA imaging have limited predictive value in our study population, and this supports the need for a management approach specific to MS. However the FRAX and Qfracture tools have been validated in large populations and may certainly have a role in identifying those patients with significant risk factors for reduced BMD independent of MS e.g. comorbid diabetes or rheumatoid arthritis. Management of patients with reduced BMD identified on DEXA scan should be in accordance with local guidelines e.g. the National Osteoporosis Guideline Group in Britain.[44]

\section{Timing of DEXA scanning}

In our study we carried out DEXA scans at a mean of 58 days after the relapse clinic assessment. This is more practical than completing DEXA imaging in the early acute stage of a relapse, as allowing some time for recovery will likely give a better reflection of long-term BMD. When interpreting DEXA results in this cohort it is also reasonable to assume that regional osteoporosis can occur in the context of a relapse, similar to that described in paretic limbs of stroke patients.[45] Areas of regional paresis should be avoided if possible during DEXA imaging. If regional osteoporosis is demonstrated in a paretic region affected by acute relapse, it may be prudent to delay a treatment decision and repeat DEXA scanning after 6 months to allow a full cycle of bone remodelling.

\section{Strengths and limitations}

The strengths of our study include the multi-modal assessment of bone status of patients (lifestyle factors, FRAX assessment, Vitamin D status and BMD), minimal exclusion criteria and our proposal of a clinical algorithm for management of bone health in this population. Our approach is novel as we 
chose to focus on a cohort of patients with active disease experiencing MS relapses. The lack of controls and the non-attendance of 10 subjects for DEXA scanning can be considered limitations. While our study reflects an average population in our relapse clinic, factors such as relapse frequency, regional and seasonal variation in Vitamin D limit the generalisability of our results across this population and across regions, so we have considered our results in the context of the relevant literature when designing our clinical algorithm.

\section{Conclusion}

Our study supports recent evidence that bone loss begins early in MS and illustrates that predicting BMD in this group is difficult. Bone loss is likely to increase with disease progression so it is necessary to identify those at risk of fractures early, treat appropriately and continue to monitor over the long-term. The algorithm proposed here should aid clinicians in early identification and management of patients at risk of fractures. While the algorithm has been designed considering our patient group (patients in an acute relapse clinic), we feel it could be applied in any similar clinical setting i.e. patients with RRMS on disease modifying medications, which could be the focus of further work. 


\section{DISCLOSURES}

Declarations/Conflicts: The authors have no declarations or conflicts relevant to the article.

Funding: Jeremy Chataway acknowledges the UK National Institute for Health Research (NIHR) University College London Hospitals/UCL Biomedical Research Centres funding scheme. 


\section{REFERENCES}

1. Bazelier MT, van Staa T, Uitdehaag BM, et al. The risk of fracture in patients with multiple sclerosis: the UK general practice research database. Journal of Bone and Mineral Research 2011;Sep;26(9):2271-2279.

2. Dennison EM, Compston JE, Flahive J, et al. Effect of co-morbidities on fracture risk: findings from the Global Longitudinal Study of Osteoporosis in Women (GLOW). Bone 2012;50(6):1288-1293.

3. Sioka C, Papakonstantinou S, Fotopoulos A, et al. Bone mineral density in ambulatory patients with multiple sclerosis. Neurological Sciences 2011;32:819-824.

4. Kurtzke JF. Rating neurologic impairment in multiple sclerosis: an expanded disability status scale (EDSS). Neurology 1983;33(11):1444-1452.

5. Zorzon M, Zivadinov R, Locatelli L, et al. Long-term effects of intravenous high dose methylprednisolone pulses on bone mineral density in patients with multiple sclerosis. European Journal of Neurology 2005;12(7):550-556.

6. Weinstock-Guttman B, Gallagher E, Baier M, et al. Risk of bone loss in men with multiple sclerosis. Multiple Sclerosis 2004;10(2):170-175.

7. Tüzün S, Altintaş A, Karacan I, Tangürek S, Saip S, Siva A. Bone status in multiple sclerosis: beyond corticosteroids. Multiple Sclerosis 2003;9(6):600-604.

8. Moen SM, Celius EG, Sandvik L, Nordsletten L, Eriksen EF, Holmøy T. Low bone mass in newly diagnosed multiple sclerosis and clinically isolated syndrome. Neurology 2011;77(2):151-157.

9. Myhr KM, Mellgren SI. Corticosteroids in the treatment of multiple sclerosis. Acta Neurologica Scandinavica Supplement 2009;(189):73-80.

10. Dovio A, Perazzolo L, Osella G, et al. Immediate fall of bone formation and transient increase of bone resorption in the course of high-dose, short-term glucocorticoid therapy in 
young patients with multiple sclerosis. The Journal of Clinical Endocrinology and Metabolism 2004;89(10):4923-4928.

11. Haugeberg G, Griffiths B, Sokoll KB, Emery P. Bone loss in patients treated with pulses of methylprednisolone is not negligible: a short term prospective observational study. Annals of the Rheumatic Diseases 2004;63(8):940-944.

12. Hearn AP, Silber E. Osteoporosis in multiple sclerosis. Multiple Sclerosis 2010;16(9):10311043

13. Kanis JA, Johnell O, Oden A, Johansson H, McCloskey E. FRAX and the assessment of fracture probability in men and women from the UK. Osteoporososis International 2008;19(4):385-397.

14. Chataway J, Porter B, Riazi A, et al. Home versus outpatient administration of intravenous steroids for multiple-sclerosis relapses: a randomised controlled trial. Lancet Neurology 2006;5(7):565-571.

15. Simon KC, Munger KL, Ascherio A. Vitamin D and multiple sclerosis: epidemiology, immunology, and genetics. Current Opinion in Neurology 2012;25(3):246-251.

16. Hippisley-Cox J, Coupland C. Derivation and validation of updated QFracture algorithm to predict risk of osteoporotic fracture in primary care in the United Kingdom: prospective open cohort study. The British Medical Journal 2012;344:3427.

17. Dobson R, Ramagopalan S, Giovannoni G. Bone health and multiple sclerosis. Multiple Sclerosis 2012;18(11):1522-1528.

18. Bazelier MT, van Staa TP, Uitdehaag BM, et al. A simple score for estimating the long-term risk of fracture in patients with multiple sclerosis. Neurology 2012;79(9):922-928.

19. McNicholas N, Patel A, Chataway J. It is better to be in a clinical trial than not: lessons learnt from clinical neurology--the management of acute multiple sclerosis relapses. $Q J M$ 2012;105(8):775-780.

20. WHO FRAX Calculator http://www.shef.ac.uk/FRAX/index.aspx (accessed 15 June 2014).

21. NHS Health Research Authority. Defining Research. http://www.hra.nhs.uk/documents/2013/09/defining-research.pdf (accessed 10 June 2014). 
22. Holick MF, Binkley NC, Bischoff-Ferrari HA, et al. Evaluation, treatment, and prevention of vitamin D deficiency: an Endocrine Society clinical practice guideline. The Journal of Clinical Endocrinology and Metabolism 2011;96(7):1911-1930. 1.

23. Hyppönen E, Power C. Hypovitaminosis D in British adults at age 45 y: nationwide cohort study of dietary and lifestyle predictors. The American Journal of Clinical Nutrition 2007;85(3):860-868.

24. Webb AR, Kift R, Durkin MT, et al. The role of sunlight exposure in determining the vitamin D status of the U.K. white adult population. British Journal of Dermatology 2010;163(5):1050-1055.

25. Munger KL, Levin LI, Hollis BW, Howard NS, Ascherio A. Serum 25-hydroxyvitamin D levels and risk of multiple sclerosis. Journal of the American Medical Association 2006;296(23):2832-2838.

26. Romagnoli E, Pepe J, Piemonte S, Cipriani C, Minisola S. Value and limitations of assessing vitamin D nutritional status and advised levels of vitamin D supplementations. European Journal of Endocrinology 2013;169(4):59-69.

27. Kirbas A, Kirbas S, Anlar O, Turkyilmaz AK, Cure MC, Efe H. Investigation of the relationship between vitamin $\mathrm{D}$ and bone mineral density in newly diagnosed multiple sclerosis. Acta Neurologica Belgica 2013;113(1):43-47.

28. Moen SM, Celius EG, Sandvik L, et al. Bone turnover and metabolism in patients with early multiple sclerosis and prevalent bone mass deficit: a population-based case-control study. PLoS One 2012;7(9):e45703.

29. Polzer K, Joosten L, Gasser J, et al. Interleukin-1 is essential for systemic inflammatory bone loss. Annals of the Rheumatic Diseases. 2010 Jan;69(1):284-290.

30. Ascherio A. Environmental factors in multiple sclerosis. Expert Review of Neurotherapeutics 2013;13(12 Suppl):3-9.

31. Bischoff-Ferrari HA, Kiel DP, Dawson-Hughes B, et al. Dietary calcium and serum 25hydroxyvitamin D status in relation to BMD among U.S. adults. Journal of Bone and Mineral Research. 2009 May;24(5):935-942. 
32. American College of Rheumatology Ad Hoc Committee on Glucocorticoid-Induced Osteoporosis. Recommendations for the prevention and treatment of glucocorticoid-induced osteoporosis: 2001 update. Arthritis \& Rheumatology 2001;44(7):1496-1503.

33. Canalis E, Mazziotti G, Giustina A, Bilezikian JP. Glucocorticoid-induced osteoporosis: pathophysiology and therapy. Osteoporososis International 2007;18(10):1319-1328.

34. K. Hayashi, M. Yamamoto, Y. Murakawa et al. Bone fragility in male glucocorticoid induced osteoporosis is not defined by bone mineral density. Osteoporososis International 2009;20(11):1889-1894.

35. B.B. Kalpakcioglu, K. Engelke, H.K. Genant. Advanced imaging assessment of bone fragility in glucocorticoid induced osteoporosis. Bone 2011;48(6):1221-1231.

36. Sorensen PS, Mellgren SI, Svenningsson A,et al. NORdic trial of oral Methylprednisolone as add-on therapy to Interferon beta-1a for treatment of relapsing-remitting Multiple Sclerosis (NORMIMS study): a randomised, placebo-controlled trial. Lancet Neurology 2009;8(6):519529.

37. Bischoff-Ferrari HA. The 25-hydroxyvitamin D threshold for better health. The Journal of Steroid Biochemistry and Molecular Biology 2007;103(3-5):614-619.

38. Dawson-Hughes B, Heaney RP, Holick MF et al. Estimates of optimal vitamin D status. Osteoporososis International 2005;16(7):713-6.

39. Hathcock JN, Shao A, Vieth R, Heaney R. Risk assessment for vitamin D. The American Journal of Clinical Nutrition 2007;85(1):6-18.

40. Dobson R, Yarnall A, Noyce AJ, Giovannoni G. Bone health in chronic neurological diseases: a focus on Multiple sclerosis and Parkinsonian syndromes. Practical Neurology 2013;13(2):70-79.

41. National Osteoporosis Society. Vitamin D and Bone Health: A Practical Clinical Guideline for Patient Management. http://www.nos.org.uk/document.doc?id=1088 (accessed 5 May 2014).

42. Sempos CT, Durazo-Arvizu RA, Dawson-Hughes B, et al. Is there a reverse J-shaped association between 25-hydroxyvitamin D and all-cause mortality? Results from the U.S. 
nationally representative NHANES. The Journal of Clinical Endocrinology and Metabolism. 2013;98(7):3001-3009.

43. Huda W, Morin RL. Patient doses in bone mineral densitometry. The British Journal of Radiology 1996;69(821):422-425.

44. National Osteoporosis Guideline Group. Osteoporosis: Clinical guideline for prevention and treatment. NOGG 2013.

45. Poole KE, Reeve J, Warburton EA. Falls, fractures, and osteoporosis after stroke: time to think about protection? Stroke 2002;33(5):1432-1436 


\section{TABLES AND FIGURES}

Table 1: Demographic Characteristics of the Population

Table 2: Bone Mineral Density of the Population

Table 3: Demographics of the group, comparing individuals with a normal DEXA scan to those with at least one measurement indicating osteopenia/osteoporosis

Figure 1: Suggested clinical algorithm for management of bone health 